\title{
Heuristic Evaluation of the User Interface for a Semi-Autonomous Agricultural Robot Sprayer
}

George Adamides

Rural Development Department, Agricultural Research Institute, Nicosia, Cyprus

\begin{abstract}
This study presents the heuristic evaluation, as a usability inspection method for Human-Robot Interaction (HRI) systems. First, the methodology to engineer a semi-autonomous agricultural robot sprayer is presented, and then findings from heuristic usability evaluation studies that were carried out on a human-robot interface for a semi-autonomous agricultural vineyard robot sprayer. The following research-based heuristics for the design of robot teleoperation were used: Platform architecture and scalability, Error prevention and recovery, Visual design, Information presentation, Robot state awareness, Interaction effectiveness and efficiency, Robot environment/surroundings awareness, and Cognitive factors. In each evaluation study, usability problems were identified, and specific suggestions were documented for HRI usability improvement. In each design iteration, a smaller number of usability issues were identified.. Results of the final heuristic evaluation showed that the system is at a good level of usability and is expected to provide satisfactory services to its typical users.
\end{abstract}

\section{Keywords}

Agricultural robot, human-robot interaction, heuristic evaluation, usability study.

Adamides, G. (2020) "Heuristic Evaluation of the User Interface for a Semi-Autonomous Agricultural Robot Sprayer", AGRIS on-line Papers in Economics and Informatics, Vol. 12, No. 3, pp. 3-12. ISSN 1804-1930. DOI 10.7160/aol.2020.120301.

\section{Introduction}

According to the Food and Agriculture organization of the United Nations (FAO), the world population will reach over 9 billion by 2050 (FAO, 2009). This projection implies that agricultural food production will need to double, in order to achieve food security. In addition, climate change, limited land and water resources (FAO, 2010), the observed shortage of agricultural workers (Hertz and Zahniser, 2013), and farmers' aging coupled with the hardness of agricultural tasks, increases the burden of producing more agricultural products, with limited resources and environmental constraints.

Due to technological advancements in recent years, robotics has begun to play a major role in our daily lives (Arad et al., 2020). Automation in agriculture, mechanization and agricultural engineering, has been a major force for increased productivity in the 20th century (McNulty and Grace, 2009). In fact, according to Huffman and Evenson (2001), the aggregate United States farm output was 5.5 times larger in 1990 than a hundred years ago.

In the past two decades, research and development on agricultural robots has been spurred on by the emergence of new and increasingly cost-effective advances in engineering, sensing and actuating technologies, along with the decrease of technology cost (Sistler, 1987). According to Adamides (2016), "robotics for agriculture is considered the domain of field systems able to perform coordinated, mechatronic actions, on the basis of processing of information acquired through camera(s) and other sensor technology, with the aim to support farmers in performing agricultural tasks."

Agriculture is a suitable application area for robotics given the hard working conditions (e.g. physical work and severe weather conditions) and difficulty and repetitiveness of the work. Robotic technology can enhance the farmer's capabilities (i.e., perception, decision-making) to carry out repetitive, tedious, and in some cases dangerous agricultural tasks (e.g. weeding (Kargar et al., 2013) and spraying (Oberti et al., 2016)) in dynamic and unstructured environments.

Unlike the case of industrial robots, which operate in a structured and controlled environment, fully 
autonomous agricultural robots are expected to operate under several complexities as identified by Edan et al. (2009). Examples of such difficulties is moving on unstructured terrain, dealing with highly variable fruits that differ in size, color (even at the same plant), and environmental issues like shading and lighting. Autonomous robotic sprayers have been developed for weed control in field applications (Åstrand and Baerveldt, 2002, Kargar et al., 2013), trees in orchards (Endalew et al., 2011, Brown et al., 2008), and vineyards (Berenstein and Edan, 2012, Berenstein and Edan, 2017). Selective spraying pesticides towards the targets, using a robot sprayer could reduce up to $30 \%$ of the pesticide (spraying material) while detecting and spraying $90 \%$ of the grape clusters (Berenstein et al., 2010). Today, vineyard spraying is achieved by spraying uniform amounts of pesticides along the vineyard rows without considering low density foliage, which requires less pesticide, or gaps between the trees. Figure 1, illustrates current methods used for spraying vineyards.

A semi-autonomous robot implies that some operations the robot carries out autonomously (as preprogrammed), while others are carried out under the supervision/ guidance of a human operator. In this sense, Fong et al. (2001) explains that human-robot interaction (HRI) is "the study of the humans, robots and the ways they influence each other." The goal of HRI in an agricultural environment is to develop and use efficiently robots such that farmers become more productive, effective, in a safe environment, freeing them from the hardness, routine and dangerous tasks (e.g. spraying pesticides).

Semi-autonomous teleoperation of an agricultural robotic system can help to effectively manage the complexity and performance limitations that current autonomous robots face due to the dynamic and unstructured agricultural environment. Semiautonomous teleoperation implies the existence of a user interface (UI) that supports human-robot interaction. Such a user interface needs to meet specific non-functional requirements, such as reliability, efficiency and usability.

This study focuses on usability, which refers to whether a HRI system can be used with interaction effectiveness, interaction efficiency, and satisfaction with which specified types of users achieve specified goals in a particular context of use. In specific, this article presents findings from heuristic usability evaluations that were carried out on two versions of such a human-robot interface for a semi-autonomous agricultural vineyard robot sprayer. Three iterations of the user-centered design process were followed to ensure the usability of the final product.

Nielsen and Molich (1990) explains that "Heuristic evaluation is a 'discount usability engineering' method for evaluating user interfaces to find their

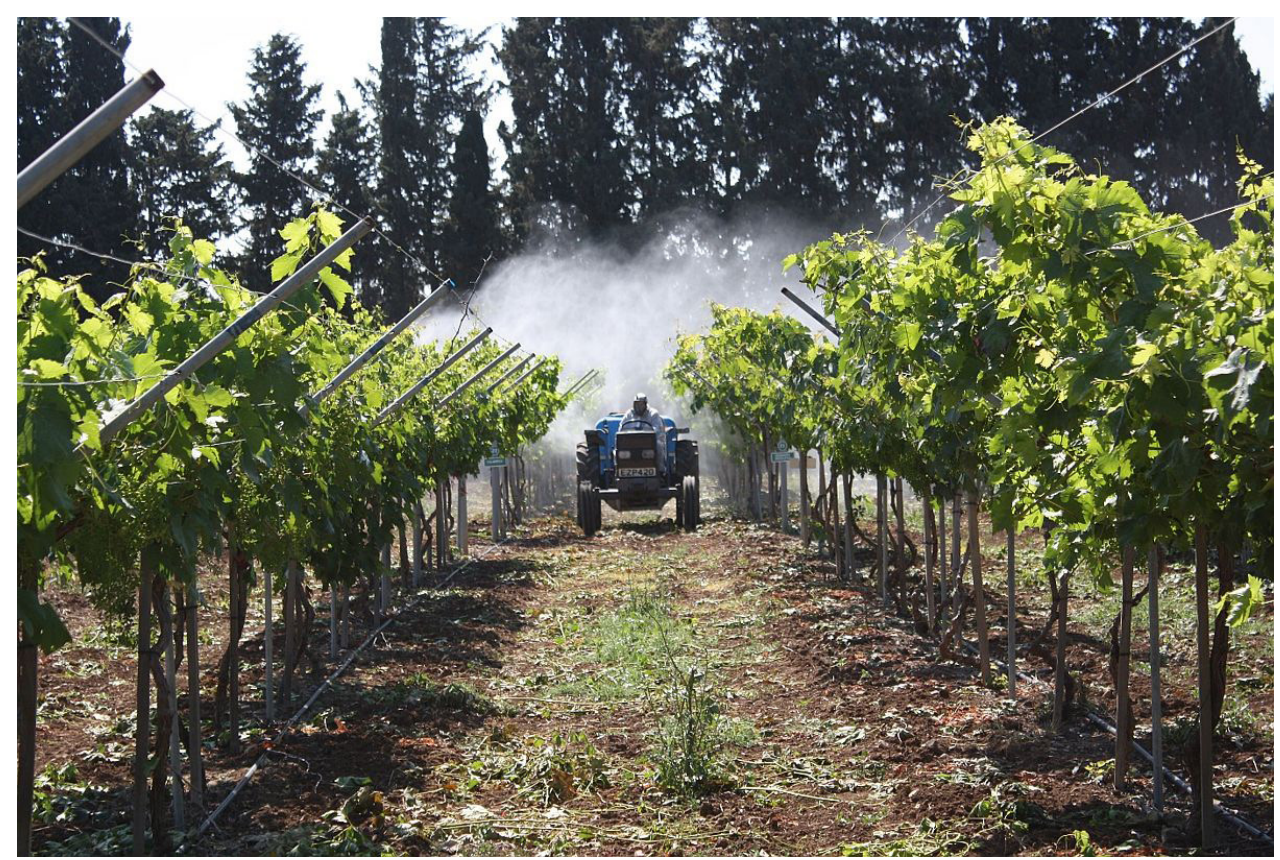

Source: author

Figure 1: Farmer on a tractor-sprayer in a vineyard field. 
usability problems". This method is characterized as "discount" because a small number of evaluators, usually 3 to 7 , suffice to reliably evaluate the usability of a user interface against a list of heuristics (the usability principles).

A usability issue is anything that can affect the user experience in a negative way. There are many sources of data that can be used to derive usability issues; the most common ones (Tullis and Albert, 2008) include user performance data (e.g., task success rate, time on task), verbal expressions of confusion or dissatisfaction (such as from a think-aloud protocol (Alan et al., 2004)), behavioral/physiological data (e.g., from eye-tracking (Poole and Linden, 2005)) and reports from usability experts (e.g. heuristic evaluation (Nielsen, 1994c)). Usability issues are often prioritized based on severity schemes (Nielsen, 1994b, Dumas and Redish, 1999) that take into account various factors (e.g. expected impact on user experience, predicted frequency of occurrence, expected impact on business goals) in an attempt to increase their usefulness for the next design iteration.

The objective of this article is twofold: a) to present the methodology to engineer a semi-autonomous agricultural robot sprayer and b) the usability evaluation of the developed user interfaces using heuristics. What follows is the presentation of the research methodology, and later results and findings are discussed. Finally, the conclusions and prospects for further research are presented.

\section{Materials and methods}

\section{Robot sprayer development stages}

A mobile platform by Robotnik (http://www. robotnik.eu) was used (Figure 2a). This platform is a medium-sized, high mobility all-terrain robot, with skid-steering kinematics based on four high power motor-wheels. This specific platform was selected because it can move both indoors (i.e. greenhouse) and outdoors (i.e. agricultural field) in a variety of field applications.

The original design of the robot was based on the analysis of user contextual interviews of farmers and agronomists that pilot tested in the field an initial version of the agricultural robot sprayer (Adamides et al., 2014). Initially, in robot version 1 (Figure 2b), several HRI related limitations were identified such as: a) the lack of peripheral vision, b) the operator required a significant amount of time to pan-tilt zoom-in and zoom-out from the main robot camera, c) limitations to Bluetooth connection via the PS3 gamepad controller, and d) illumination of the laptop monitor (used for robot control) due to sunlight. Following, informal interviews and documentation of the pilot participants' observations, several modifications on the platform resulted to an improved version.

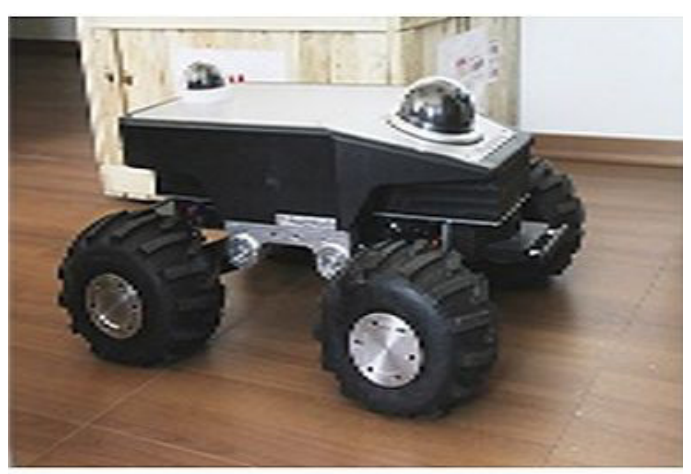

(a)

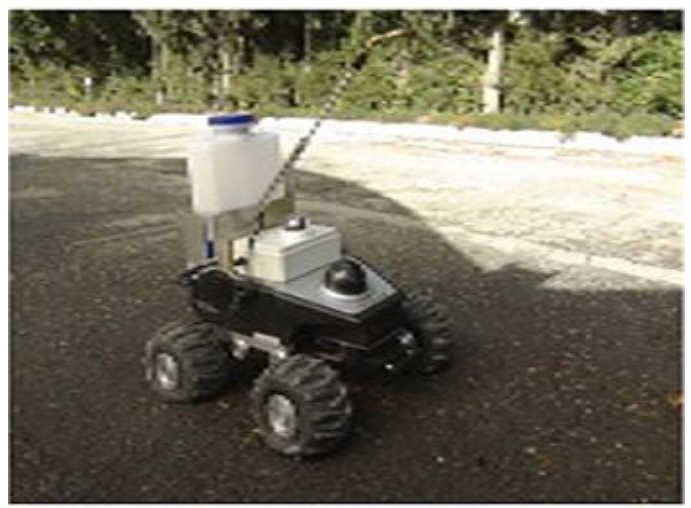

(b)

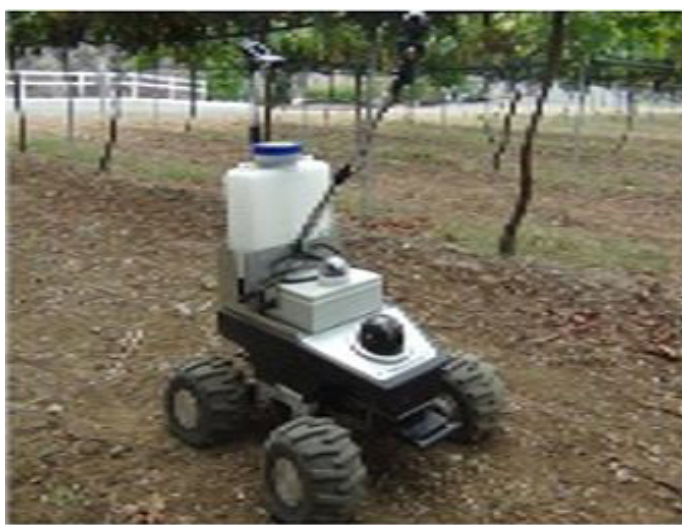

(c)

Source: author

Figure 2: Development stages of the semi-autonomous robot sprayer.

The final version (Figure 2c) included a peripheral camera on the back-top of the platform 
and an end-effector camera on-top of the nozzle canon sprayer. In order to solve the issue of the distance limit of the PS3 gamepad controller, two solutions were provided: a) connecting the controller through WiFi and b) adding a PC keyboard alternative as input device. Similarly, to address the issue of sunlight and illumination of the PC monitor, also two alternatives were implemented: a) using digital glasses and b) teleoperating the robot from inside an office environment.

The following HRI taxonomy (Table 1) was assumed in this article for the semi-autonomous agricultural robot sprayer, based on the HRI taxonomy proposed by Yanco and Drury (2004).

In the specific case of the semi-autonomous robot sprayer, the navigation task (robot path guidance) was performed in teleoperation mode, while the target marking/ identification and spraying tasks were performed in autonomous or semi-autonomous mode.

\section{User interface heuristic evaluation}

The heuristic evaluation method was employed, as one of the most popular usability inspection techniques, which are also known as expert-based methods, user-free methods or methods performed in the lab without end-users. An adequate number of experts was found and recruited so that reliable evaluation results could be obtained. First, the evaluators were informed about the system goal, its representative users and their typical tasks and the developers' design goals and expectations. Next, they used the system and conducted an individual heuristic evaluation according to a specific protocol, a selected set of heuristics appropriate for the evaluation context, and a template for reporting the identified usability issues. The evaluators were situated at the Hellenic Open University Software Quality Assessment laboratory and controlled the robot remotely, which was located at the Open University of Cyprus, Nicosia premises. An appropriate lab-simulation environment was created, including various paths and targets. After each individual evaluation, the participating evaluators conducted a focus group to group and prioritize the identified usability issues.

Four usability experts - an adequate number to ensure reliable results (Nielsen, 1994a) - conducted a heuristic usability evaluation on two user interfaces. All four have undergraduate and/or postgraduate studies in Computer Science and extensive experience in the design and evaluation of interactive systems.

Two user interfaces for the Semi-Autonomous Agricultural Robot Sprayer were evaluated: UIv0 and UIv1 Figure 3 presents the two main screens of these user interfaces.

\begin{tabular}{|c|c|c|}
\hline Category & Description & Classification \\
\hline Task type & $\begin{array}{l}\text { There are three tasks to be executed in this HRI: } \\
\text { guiding the robot in the vineyards, identifying targets } \\
\text { to spray, and the actual spraying task }\end{array}$ & $\begin{array}{l}\text { [Navigation (robot path guidance), } \\
\text { Target Marking/ Identification, } \\
\text { Spraying] }\end{array}$ \\
\hline Task Criticality & $\begin{array}{l}\text { Given that in robot navigation there is a possibility } \\
\text { to harm either the robot or bystanders or the vines, } \\
\text { the task criticality is High. For the target } \\
\text { identification and spraying the criticality is set to low. }\end{array}$ & [High, Low] \\
\hline Robot morphology & Mobile robotic platform with spraying capabilities & [Functional] \\
\hline Ratio of people to robots & One human operator and one robot sprayer & {$[1: 1]$} \\
\hline Composition of robot teams & Same robot & [Homogeneous] \\
\hline Level of shared interaction & One human operator and one robot sprayer & [one human, one robot] \\
\hline Interaction roles & $\begin{array}{l}\text { During Autonomous mode the human is acting as } \\
\text { supervisor. During the teleoperation mode the human } \\
\text { is acting as Operator. During the semi-Autonomous } \\
\text { mode the human is acting as teammate. }\end{array}$ & [Supervisor, Operator, Teammate] \\
\hline $\begin{array}{l}\text { Type of human-robot physical } \\
\text { proximity }\end{array}$ & The human and the robot are not collocated & [Avoiding] \\
\hline Decision support for operators & Battery level, camera and sonar sensors & [Provided sensors] \\
\hline Time/Space taxonomy & $\begin{array}{l}\text { Human and robot operate at the same time } \\
\text { in different locations }\end{array}$ & $\begin{array}{l}\text { [Time (Synchronous), Space } \\
\text { (Non-collocated)] }\end{array}$ \\
\hline $\begin{array}{l}\text { Autonomy level / Amount } \\
\text { of intervention }\end{array}$ & $\begin{array}{l}\text { There is a continuum for robot control ranging } \\
\text { from teleoperation to full autonomy }\end{array}$ & [Autonomy+Intervention=100\%] \\
\hline
\end{tabular}

Source: author 


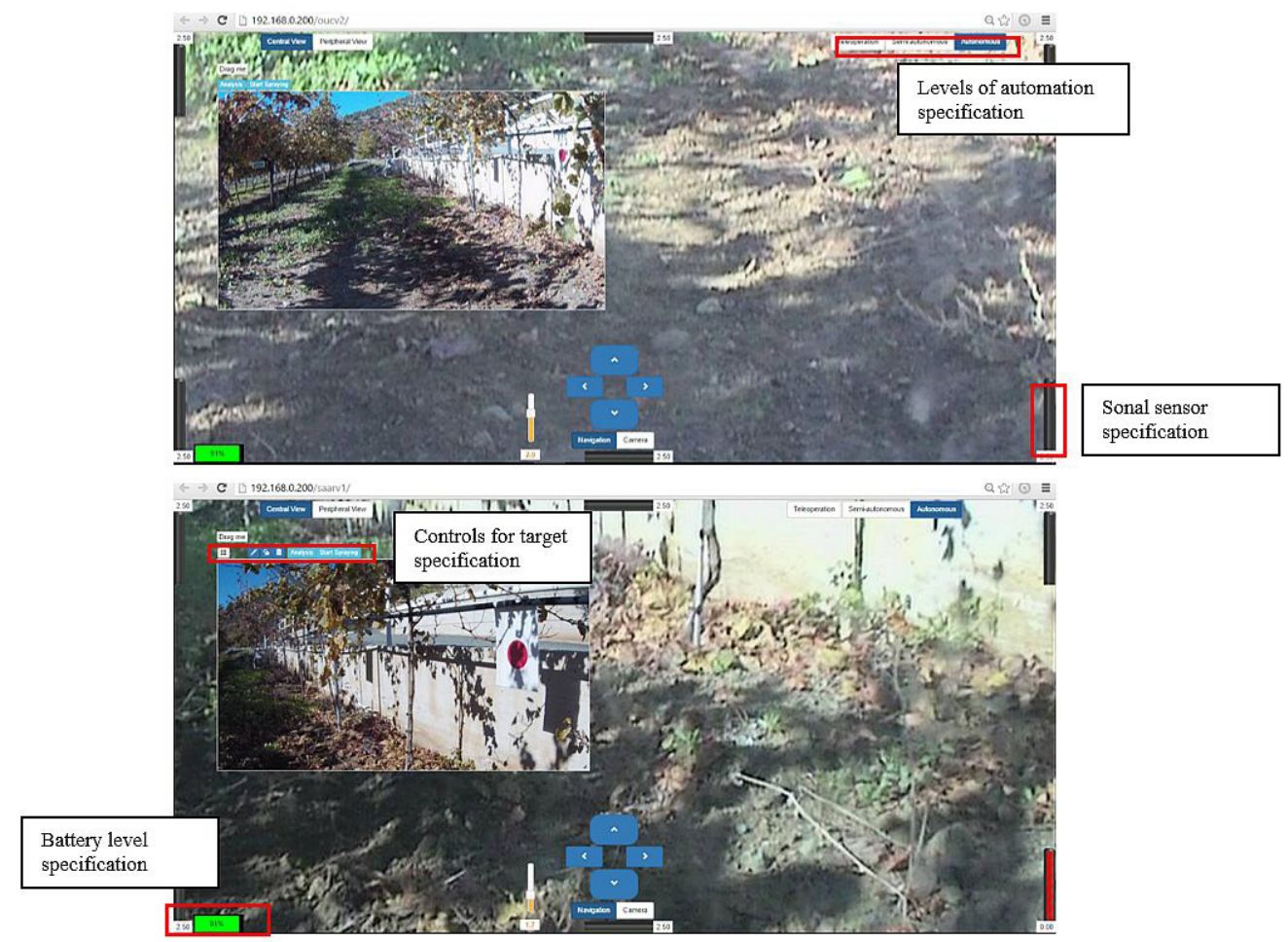

Note: The red rectangles and black text boxes are not part of each user interface Source: author

Table 1: HRI taxonomy for the semi-autonomous agricultural robot sprayer.

UIv0: In terms of functionality, the main designs considered were: a) on-screen controls of the robot movement and camera movement, b) presentation of camera views, and c) addition of elements for displaying sensor information (visual and auditory feedback) for distance from the robot sides and battery level. One important priority when designing UIv0, was to enable the operator to use the entire screen and support interaction through either the keyboard or the mouse.

UIv1: is an upgraded user interface of UIv0. It provides functionality for target pointing. In specific, UIv1 supports both manual (user points to targets) and automated target specification through a pattern recognition algorithm.

The same procedure was followed in all heuristic evaluation studies. A set of research-based heuristics for the design of robot teleoperation, which have been developed in Adamides et al. (2015) were used. These are:

- Platform architecture and scalability: The user interface of an HRI system should be flexible to follow and benefit from developments in computing and robotic technologies.

- Error prevention and recovery:
The information provided by the user interface should prevent user errors, and if a user makes a mistake, the user interface should allow for its rectification. In contrast with undoing a "Cut" operation in a word processor, a "Cut" command to prune a tree through a teleoperated AgriRobot cannot be undone.

- Visual design: Since the user interface is the communication medium between the operator and the remote robot, it should provide the operator with only relevant information (from video and other robot sensors) in a simple, consistent, effective, and minimalist way. Specific examples include minimizing use of multiple windows, avoiding window occlusion, providing large video windows and displaying the robot's body in the interface.

- Information presentation: Controlling a remotely located robot is demanding on operators who need to integrate various sources of information coming from the robot cameras and sensors. Therefore, information presentation is of high importance in this type of user interface designs, to enhance situation awareness of the operators, and to bridge 
the gaps of execution and evaluation (Alan et al., 2004).

- Robot state awareness: The robot should be able to self-inspect its systems and take autonomous action or ask for user intervention. The human operator should have a clear understanding about the robot status and activities. For instance, to support understanding of the camera(s) and their position(s), the over-all mission and the current progress, and when multiple robots are available, use one to view another.

- Interaction effectiveness and efficiency: In HRI, efficiency is measured in terms of the time required to complete a task; effectiveness is measured in terms of how well a task is completed.

- Robot environment/surroundings awareness: Environment awareness is essential, because in field robot teleoperation it is important to have knowledge of the robot's whereabouts and the area covered, such as orientation, obstacles, or why a robot is not moving. This can be accomplished through maps, orientation information (such as compass), and sensors that will provide the necessary information about the robot's surroundings.

- Cognitive factors: Cognitive factors are characteristics that affect performance and learning. The user interface of a teleoperated HRI system should be designed such that it directs the user's attention to the task the robot is operating, improves learnability, and provides fused information from the various sensors and cameras from the robot, in order to lower the cognitive load on the user.

Next, the evaluators were informed about the system goal, its representative users and their typical tasks. Subsequently, each evaluator conducted a heuristic evaluation of the system. To this end, they were provided with access to the semiautonomous agricultural robot sprayer UI versions under evaluation. They first familiarized themselves with the system by performing typical user tasks and exploring its functionality. Next, they inspected the system, identified usability issues and wrote them down following specific evaluation template. For each problem, they noted the heuristic violated and rated its severity on a scale from 1 to $5(1=$ a little important, it does not significantly affect the user interaction, 5=extremely important, catastrophic problem that may result in unsuccessful task, danger to life or damage to property). In evaluating the severity of a usability problem, they were asked to take into account the following factors (Nielsen, 1995): a) frequency, b) impact, and c) persistence. Finally, each evaluator was asked to provide a design suggestion for resolving the identified usability issue. The four evaluators produced individual reports with the identified usability issues per heuristic rule (Adamides et al., 2015).

After each individual evaluation, the study coordinator and the evaluators participated in a focus group in order to produce the final list of unique problems, discuss on the final severity ratings and proposals for solutions.

\section{Results and discussion}

Results of the heuristic evaluation showed that the systems under evaluation provide very good (in terms of usability issues identified by experts) services to their expected typical users. A small number of usability problems were identified, whose redress can improve the overall user experience with the system.

In the following, the results for each evaluated system are presented. The total number of expected problems for the each system was calculated using the formula (Nielsen and Landauer, 1993):

$\mathrm{N}=\frac{1-(1-\mathrm{j})^{i}}{\text { Problemsfound(i) }}$

where $N$ is the total number of expected usability problems, $i$ is the number of independent experts-evaluators, ProblemsFound( $i)$ is the total number of unique usability issues identified by the participating evaluators, and $\mathrm{j}$ is the average proportion of problems found by a single evaluator.

\section{A. First user interface: UIv0}

For UIv0, 13 usability issues were identified. Most $(77 \%)$ of these usability issues were related to violations of the following four heuristics: a) $23 \%$ were violations of heuristic 4 (Information presentation), b) $23 \%$ were violations of heuristic 5 (Robot state awareness), c) 15\% were violations of heuristic 6 (Interaction effectiveness and efficiency) and d) $15 \%$ were violations of heuristic 8 (Cognitive factors). In terms of problem severity, the issues with the highest priority were related to violations of the following three heuristics: a) heuristic 5 (Robot state awareness) with the highest average severity (4.0), b) heuristic 2 (Error prevention and recovery) 
with the second from top average severity (4.0), and c) heurist 3 (Visual design) with also second from top average severity (4.0).

The expected number of usability problems for UIv0 was calculated to 42, which is above the average number of usability problems (35) observed in a rather mature interactive system (Nielsen and Landauer, 1993). In addition, a substantial number of problems (9) were rated as $3+$ on a severity scale from 1 to 5 . The average severity of the identified problems is characterized as medium (3.3). All in all, the system is at a satisfactory level of usability. However, there are changes that could further improve its usability.

\section{B. Second user interface: UIv1}

Regarding UIv1, 10 usability issues were identified. Most $(80 \%)$ of these usability issues were related to violations of the following four heuristics: a) $20 \%$ were violations of heuristic 4 (Information presentation), b) $20 \%$ were violations of heuristic 5 (Robot state awareness), c) $20 \%$ were violations of heuristic 6 (Interaction effectiveness and efficiency) and d) $20 \%$ were violations of heuristic 8 (Cognitive factors). In terms of problem severity, the issues with the highest priority were related to violations of the following three heuristics: a) heuristic 2 (Error prevention and recovery) with the highest average severity $(4.0)$, b) heuristic 6 (Interaction effectiveness and efficiency) with the second from top average severity (3.0), and c) heuristic 5 (Robot state awareness) with third from top average severity (2.0).

The expected number of usability problems for UIv1 was calculated to 15 , which is less than half the average number of usability problems (35) observed in a rather mature interactive system. In addition, a small number of problems (3) were rated as $3+$ on a severity scale from 1 to 5 . The average severity of the identified problems is characterized as low (2.1). These findings (shown in Figure 4) tend to provide support that the system is at a good level of usability.

According to the expert evaluators, one important advantage of both user interface versions is that they take full advantage of the screen size providing a large window for the central and peripheral views. In addition, the user can easily customize the placement and size of the end-effector camera view. Furthermore, implicit switching of autonomy level is supported, but it should be better communicated to the user. Moreover, important information, such as the exact distance from obstacles and the remaining battery level, are always available. However, equally important information, such as the remaining level of spraying liquid (the robot is used to spray vineyards), is not available at all.

UIv1 supports functionality for targeted spraying in a rather intuitive way. The target identification algorithm automatically selects targets (i.e. grape clusters) to spray and the spraying is activated after 2 seconds, unless the operator interrupts this action viz. to cancel a false negative or modify a false positive target for spraying. However, there are UI improvements that could be made in the manual target addition and deletion to better reflect what the user is doing. In addition, these systems provide support

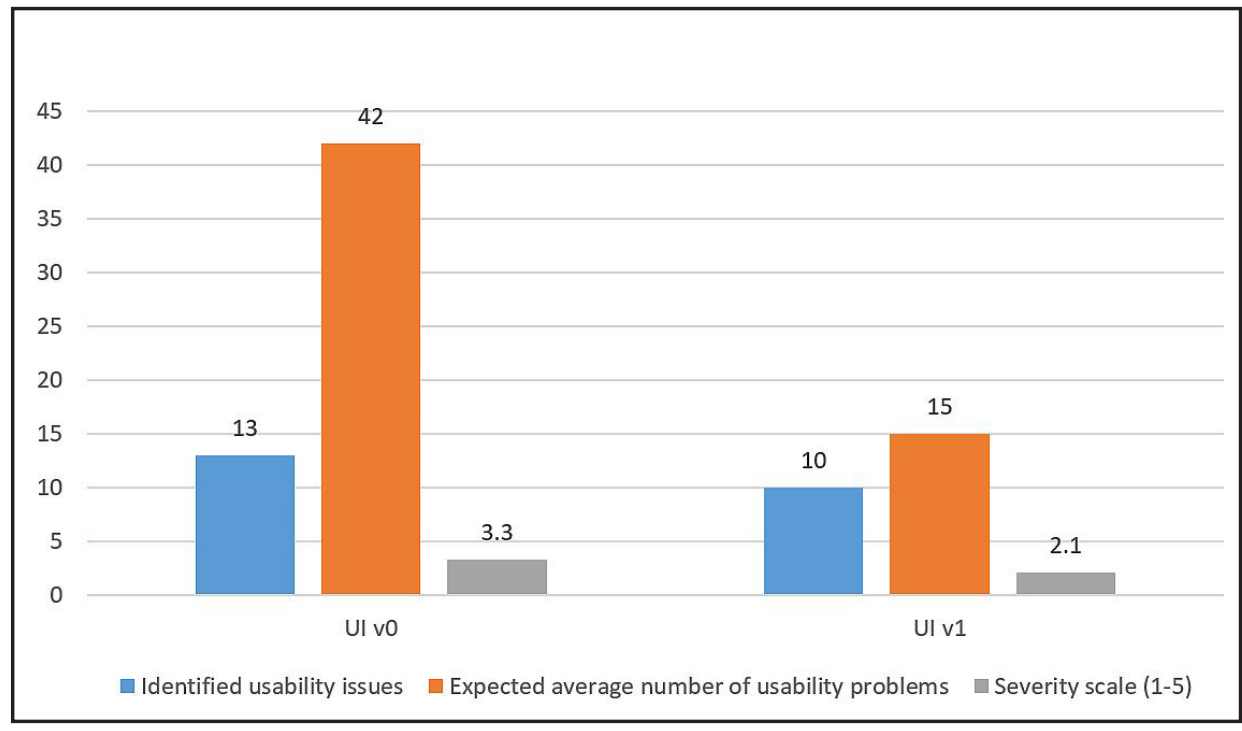

Source: author

Figure 4: Heuristic evaluation results of Uiv0 and Uiv1. 
for automated target identification, which may lead to increased efficiency in the actual field. However, the associated dialogue for changing the algorithm settings is in a highly technical and complicated language for the typical user.

\section{Conclusion}

This study presented in detail the heuristic evaluation method, and how this was applied, as a usability inspection method for Human-Robot Interaction (HRI) systems. The usability study findings provide evidence that the final version of the semi-autonomous robot sprayer system provides satisfactory services to its typical users. These advantages, combined with the increased usability of the UIv1 (final) system, may result in high adoption from its end users. This study is limited by the fact that it focused on the usability evaluation of the user interface for a HRI system. Additional experiments are underway and will focus on specific tasks such as comparing different applications of spraying (e.g. using a robotic arm) and evaluate the amount of spraying chemicals saved, in addition to other agricultural tasks that can be automated through robotization.

Corresponding authors

George Adamides

Agricultural Research Institute, P.O. Box 22016, 1516 Nicosia, Cyprus

E-mail:gadamides@ari.gov.cy

\section{References}

[1] Adamides, G. (2016) „User Interfaces for Human-Robot Interaction: Application on a SemiAutonomous Agricultural Robot Sprayer“, PhD Doctoral Dissertation, Open University of Cyprus, Nicosia. ISBN 978-9963-695-49-2.

[2] Adamides, G., Christou, G., Katsanos, C., Xenos, M. and Hadzilacos, T. (2015) „Usability Guidelines for the Design of Robot Teleoperation: A Taxonomy“, IEEE Transactions on Human-Machine Systems, Vol. 45, No. 2, pp. 256-262. ISSN 2168-2291. DOI 10.1109/thms.2014.2371048.

[3] Adamides, G., Katsanos, C., Christou, G., Xenos, M., Papadavid, G. and Hadzilacos, T. (2014) „User interface considerations for telerobotics: The case of an agricultural robot sprayer", In Second international conference on remote sensing and geoinformation of the environment (RSCy2014), Vol. 9229, pp. 92291W, International Society for Optics and Photonics. DOI 10.1117/12.2068318.

[4] Alan, D., Janet, F., Gregory, A. and Russell, B. (2004) „Human-computer interaction“, England: Pearson Education Limited. ISBN 978-0-13-046109-4.

[5] Albert, W. and Tullis, T. (2008) „Measuring the User Experience: Collecting, Analyzing, and Presenting Usability Metrics (Interactive Technologies)“, Morgan Kaufmann. E-ISBN 978-0-12-415781-1.

[6] Arad, B., Balendonck, J., Barth, R., Ben-Shahar, O., Edan, Y., Hellström, T., Hemming, J., Kurtser, P., Ringdahl, O., Tielen, T. and van Tuijl, B. (2020) „Development of a sweet pepper harvesting robot", Journal of Field Robotics, Vol. 34, No. 6, pp. 1027-1039. E-ISSN 1556-4967. DOI 10.1002/rob.21937.

[7] Åstrand, B. and Baerveldt, A.-J. (2002) „An Agricultural Mobile Robot with VisionBased Perception for Mechanical Weed Control“, Autonomous Robots, Vol. 13, pp. 21-35. E-ISSN 1573-7527, ISSN 0929-5593. DOI 10.1023/a:1015674004201.

[8] Berenstein, R. and Edan, Y. (2012), Evaluation of marking techniques for a human-robot selective vineyard sprayer", Paper presented at the International Conference of Agricultural Engineering, Valencia, Spain.

[9] Berenstein, R. and Edan, Y. (2017) „Human-robot collaborative site-specific sprayer“, Journal of Field Robotics, Vol. 34, No. 8, pp. 1519-1530. E-ISSN 1556-4967. DOI 10.1002/rob.21730.

[10] Berenstein, R., Shahar, O. B., Shapiro, A. and Edan, Y. (2010) „Grape clusters and foliage detection algorithms for autonomous selective vineyard sprayer", Intelligent Service Robotics, Vol. 3, No. 4, pp. 233-243. E-ISSN 1861-2784, ISSN 1861-2776. DOI 10.1007/s11370-010-0078-z. 
[11] Brown, D., Giles, D., Oliver, M. and Klassen, P. (2008) „Targeted spray technology to reduce pesticide in runoff from dormant orchards“, Crop Protection, Vol. 27, pp. 545-552. ISSN 0261-2194. DOI 10.1016/j.cropro.2007.08.012.

[12] Dumas, J. S. and Redish, J. C. (1999) „A practical guide to usability testing“, Intellect Books. ISBN 1-84150-020-8.

[13] Edan, Y., Han, S. and Kondo, N. (2009) „Automation in Agriculture“, Springer Handbook of Automation. Berlin, Heidelberg: Springer Berlin Heidelberg. pp. 1095-1128. ISBN 10: 3540788301, ISBN 13: 9783540788300. DOI 10.1007/978-3-540-78831-7_63.

[14] Endalew, A. M., Debaer, C., Rutten, N., Vercammen, J., Delele, M. A., Ramon, H., Nicolaï, B. M. and Verboven, P. (2011) „Modelling the effect of tree foliage on sprayer airflow in orchards“, Boundary-Layer Meteorology, Vol. 138, No. 1, pp. 139-162. E-ISSN 1573-1472, ISSN 0006-8314. DOI 10.1007/s10546-010-9544-6.

[15] FAO (2009) „How to Feed the World in 2050“, Population and Development Review, Vol. 35, No. 4, pp. 837-839. E-ISSN ISSN:1728-4457. DOI 10.1111/j.1728-4457.2009.00312.x.

[16] FAO (2010) „Climate-smart Agriculture: Policies, Practices and Financing for Food Security, Adaptation and Mitigation“, Food and Agriculture Organization of the United Nations, Rome, Italy.

[17] Fong, T., Thorpe, C. and Baur, C. (2001) „Collaboration, dialogue, human-robot interaction“, In: Jarvis R. A., Zelinsky A. (eds) Robotics Research, Springer Tracts in Advanced Robotics, Vol 6. Springer, Berlin, Heidelberg. DOI 10.1007/3-540-36460-9_17.

[18] Hertz, T. and Zahniser, S. (2013) „Is there a farm labor shortage?“, American Journal of Agricultural Economics, Vol. 95, No. 2, pp. 476-481. E-ISSN 1467-8276, ISSN 0002-9092. DOI 10.1093/ajae/aas090.

[19] Huffman, W. E. and Evenson, R. E. (2001) „Structural and productivity change in US agriculture, 1950-1982“, Agricultural economics, Vol. 24, No. 2, pp. 127-147. ISSN 0169-5150. DOI 10.1111/j.1574-0862.2001.tb00019.x.

[20] Kargar, B., Amir, H. and Shirzadifar, A. M. (2013) „Automatic weed detection system and smart herbicide sprayer robot for corn fields", Paper presented at the First RSI/ISM International Conference on Robotics and Mechatronics. DOI 10.1109/ICRoM.2013.6510152.

[21] McNulty, P. B. and Grace, P. M. (2009) „Agricultural Mechanization and Automation“, In J. K. Schueller (Ed.), Encyclopedia of Life Support Systems (EOLSS), Vol. I: Eolss Publishers Company Limited. E-ISBN 978-1-84826-096-2, ISBN 978-1-84826-546-2. DOI 10.13140/2.1.4421.9683.

[22] Nielsen, J. (1994a) „How to conduct a heuristic evaluation“, Nielsen Norman Group, 1, pp. 1-8.

[23] Nielsen, J. (1994b) „Usability engineering“, Morgan Kaufmann. ISBN 978-0125184069.

[24] Nielsen, J. (1994c) „Usability inspection methods“, In Conference companion on Human factors in computing systems, pp. 413-414. DOI 10.1145/259963.260531.

[25] Nielsen, J. (1995) „Severity ratings for usability problems“,Papers and Essays. [Online]. Available: http://www.useit.com/papers/heuristic/severityrating.html [Accessed: 23 June, 2020].

[26] Nielsen, J. and Landauer, T. K. (1993) „A mathematical model of the finding of usability problems“, Paper presented at the Proceedings of the INTERACT'93 and CHI '93 conference on Human factors in computing systems, pp. 206-213. ISBN 978-0-89791-575-5. DOI 10.1145/169059.169166.

[27] Nielsen, J. and Molich, R. (1990) „Heuristic evaluation of user interfaces“, Paper presented at the Proceedings of the SIGCHI conference on Human factors in computing systems: Empowering people. ISBN 978-1-4503-0228-9. DOI 10.1145/97243.97281.

[28] Oberti, R., Marchi, M., Tirelli, P., Calcante, A., Iriti, M., Tona, E., Hočevar, M., Baur, J., Pfaff, J., Schütz, Ch. and Ulbrich, H. (2016) „Selective spraying of grapevines for disease control using a modular agricultural robot“, Biosystems Engineering, Vol. 146, pp. 203-215. ISSN 1537-5110. DOI 10.1016/j.biosystemseng.2015.12.004. 
[29] Poole, A. and Linden, B. (2005) „Eye Tracking in Human-Computer Interaction and Usability Research: Current Status and Future“, In C. Ghaoui (Ed.), Encyclopedia of human computer interaction: IGI Global. E-ISBN13: 9781591407980, ISBN13: 9781591405627, ISBN10: 1591405629. DOI 10.4018/978-1-59140-562-7.ch034.

[30] Sistler, F. E. (1987) „Robotics and intelligent machines in agriculture“, IEEE Journal of Robotics and Automation, Vol. 3, No. 1, pp. 3-6. ISSN 0882-4967. DOI 10.1109/JRA.1987.1087074.

[31] Yanco, H. A. and Drury, J. (2004) „Classifying human-robot interaction: an updated taxonomy“, In 2004 IEEE International Conference on Systems, Man and Cybernetics (IEEE Cat. No. 04CH37583), Vol. 3, pp. 2841-2846. ISBN 0-7803-8566-7, ISSN 1062-922X. DOI 10.1109/ICSMC.2004.1400763. 